\title{
Formation of dust-rich planetesimals from sublimated pebbles inside of the snow line
}

\author{
S. Ida ${ }^{1}$ and T. Guillot ${ }^{2}$ \\ ${ }^{1}$ Earth-Life Science Institute, Tokyo Institute of Technology, Meguro-ku, Tokyo 152-8550, Japan \\ e-mail: ida@elsi.jp \\ 2 Laboratoire J.-L. Lagrange, Université Côte d'Azur, Observatoire de la Côte d'Azur, CNRS, 06304 Nice, France \\ Received 10 September 2016 / Accepted 2 November 2016
}

\begin{abstract}
Context. For up to a few millions of years, pebbles must provide a quasi-steady inflow of solids from the outer parts of protoplanetary disks to their inner regions.

Aims. We wish to understand how a significant fraction of the pebbles grows into planetesimals instead of being lost to the host star. Methods. We examined analytically how the inward flow of pebbles is affected by the snow line and under which conditions dust-rich (rocky) planetesimals form. When calculating the inward drift of solids that is due to gas drag, we included the back-reaction of the gas to the motion of the solids.

Results. We show that in low-viscosity protoplanetary disks (with a monotonous surface density similar to that of the minimum-mass solar nebula), the flow of pebbles does not usually reach the required surface density to form planetesimals by streaming instability. We show, however, that if the pebble-to-gas-mass flux exceeds a critical value, no steady solution can be found for the solid-to-gas ratio. This is particularly important for low-viscosity disks $\left(\alpha<10^{-3}\right)$ where we show that inside of the snow line, silicate-dust grains ejected from sublimating pebbles can accumulate, eventually leading to the formation of dust-rich planetesimals directly by gravitational instability.

Conclusions. This formation of dust-rich planetesimals may occur for extended periods of time, while the snow line sweeps from several au to inside of $1 \mathrm{au}$. The rock-to-ice ratio may thus be globally significantly higher in planetesimals and planets than in the central star.
\end{abstract}

Key words. planets and satellites: formation - planet-disk interactions - accretion, accretion disks

\section{Introduction}

Determining the fate of solids in protoplanetary disks is key for understanding the birth and growth of planets and planetary systems. While small grains are coupled to the disk gas, large particles drift inward as a consequence of angular momentum loss by aerodynamical gas drag. For meter sizes (assuming compact grains), the inward drift velocity is $\sim 10^{-2} \mathrm{au} / \mathrm{yr}$ (e.g., Weidenschilling 1980; Nakagawa et al. 1981). For small dust grains, growth through pairwise collisions is faster than drift so that they grow in situ until they reach 1 to $100 \mathrm{~cm}$, at which point drift starts to dominate (e.g., Okuzumi et al. 2012; Lambrechts \& Johansen 2014). These so-called pebbles then drift rapidly with limited growth, implying that without a mechanism to suppress the drift, they would be lost to the central star.

Planetesimals would form directly by gravitational instability (GI) in the dust disk if it is sufficiently thin and dense (e.g., Goldreich \& Ward 1973). However, even in low-turbulence disks, the Kelvin-Helmholtz (KH) instability generated by the vertical shear between the dust subdisk and the gas prevents the development of a disk that is thin enough (e.g., Weidenschilling 1995; Sekiya 1998). Youdin \& Shu (2002) proposed that migrating dust (or pebbles) would pile up in the inner disk to become gravitationally unstable (see also Laibe et al. 2012), but they neglected grain growth, which was then shown to prevent this pile-up (see Krijt et al. 2016).

Another possibility to form planetesimals is to invoke streaming instabilities (SI) in the drifting pebble flow: when their density is high enough, clumps can form, and because they undergo relatively less gas drag, they accrete individual pebbles to rapidly form planetesimals of 100 to $1000 \mathrm{~km}$ (Youdin \& Goodman 2005; Johansen et al. 2007). However, this mechanism requires a high solid-to-gas ratio $(Z)$ and has also been shown to be difficult to achieve in realistic disks (Krijt et al. 2016).

With analytical calculations, we examine here the formation of planetesimals from drifting pebbles in smooth disks (i.e., without pressure bumps, gaps, or vortexes) through these two mechanisms. We highlight the importance of sublimation across the snow line. Instead of examining the consequence of ice deposition beyond the snow line in turbulent disks (e.g., Stevenson \& Lunine 1988; Ros \& Johansen 2013; Armitage et al. 2016), following Saito \& Sirono (2011), we concentrate on the region inside the snow line where dust grains ejected from sublimated pebbles are present.

\section{Pebble-to-gas surface density ratio}

We consider a protoplanetary disk characterized by a steady gas accretion rate $\dot{M}_{*}$ in which the solids are in the form of pebbles migrating inward at a mass flux $\dot{M}_{\text {peb }}$. The surface density of the migrating pebbles $\Sigma_{\mathrm{p}}$ and the disk gas $\Sigma_{\mathrm{g}}$ are given by

$$
\begin{aligned}
& \Sigma_{\mathrm{p}}=\dot{M}_{\mathrm{peb}} / 2 \pi r v_{r}, \\
& \Sigma_{\mathrm{g}} \simeq \dot{M}_{*} / 3 \pi v \simeq \dot{M}_{*} / 3 \pi \alpha h_{\mathrm{g}}^{2} \Omega_{\mathrm{K}},
\end{aligned}
$$


where $v$ is the turbulent viscosity of the gas disk, $h_{\mathrm{g}}$ the gas scale height, $\Omega_{\mathrm{K}}$ the Keplerian frequency, and $v_{r}$ is the pebble migration speed. We use the $\alpha$-prescription, that is, $v \simeq \alpha h_{\mathrm{g}}^{2} \Omega_{\mathrm{K}}$.

The inward radial drift speed of solids was calculated in the limit of a static disk by Nakagawa et al. (1986) and in the limit of a low solid-to-gas ratio by Guillot et al. (2014). Combining the two yields

$v_{r}=-\Lambda^{2} \frac{2 \tau_{\mathrm{s}}}{1+\Lambda^{2} \tau_{\mathrm{s}}^{2}} \eta v_{\mathrm{K}}+\Lambda \frac{1}{1+\Lambda^{2} \tau_{\mathrm{s}}^{2}} u_{v}$,

where the back-reaction of the gas to the motion of solids has been included through $\Lambda \equiv \rho_{\mathrm{g}} /\left(\rho_{\mathrm{g}}+\rho_{\mathrm{p}}\right)$, and $\rho_{\mathrm{g}}$ and $\rho_{\mathrm{p}}$ are the mid-plane densities of gas and solids, respectively. We included the $\Lambda$-dependence of $u_{v}$ as well for later purposes. In Eq. (2), the size of the solids is defined through their Stokes number $\tau_{\mathrm{s}}$, which is the ratio of their stopping time due to gas drag $\left(t_{\text {stop }}\right)$ to the Kepler frequency as

$\tau_{\mathrm{s}}=t_{\mathrm{stop}} \Omega_{\mathrm{K}}$,

$u_{v}$ is the radial velocity of the accreting disk gas, which in the inner regions of a vertically uniform disk may be approximated by

$u_{v} \sim-3 v / 2 r \sim-3 \alpha h_{\mathrm{g}}^{2} \Omega_{\mathrm{K}} / 2 r \sim-(3 / 2) \alpha\left(h_{\mathrm{g}} / r\right)^{2} v_{\mathrm{K}}$,

and $\eta(\ll 1)$ is the deviation fraction of the gas orbital angular velocity $(\Omega)$ relative to the Keplerian angular velocity $\left(\Omega_{\mathrm{K}}\right)$ that is due to the radial pressure gradient in the disk,

$\eta=\frac{\Omega_{\mathrm{K}}-\Omega}{\Omega_{\mathrm{K}}} \simeq \frac{1}{2}\left(\frac{h_{\mathrm{g}}}{r}\right)^{2}\left(-\frac{\mathrm{d} \ln P}{\mathrm{~d} \ln r}\right)$.

From Eqs. (2), (4), and (5),

$v_{r} \simeq-\frac{1}{1+\Lambda^{2} \tau_{\mathrm{s}}^{2}}\left[\Lambda^{2} \tau_{\mathrm{s}}\left(-\frac{\mathrm{d} \ln P}{\mathrm{~d} \ln r}\right)+\Lambda \frac{3 \alpha}{2}\right]\left(\frac{h_{\mathrm{g}}}{r}\right)^{2} v_{\mathrm{K}}$.

Using Eq. (1), we then obtain the solid-to-gas ratio as

$$
\begin{aligned}
Z=\frac{\Sigma_{\mathrm{p}}}{\Sigma_{g}} & \simeq \frac{3 \alpha}{2}\left(\frac{h_{\mathrm{g}}}{r}\right)^{2} \frac{v_{\mathrm{K}}}{v_{r}} \frac{\dot{M}_{\mathrm{peb}}}{\dot{M}_{*}} \\
& \simeq\left(1+\Lambda^{2} \tau_{\mathrm{s}}^{2}\right)\left[\frac{2 \tau_{\mathrm{s}}}{3 \alpha} \Lambda^{2}\left(-\frac{\mathrm{d} \ln P}{\mathrm{~d} \ln r}\right)+\Lambda\right]^{-1} \frac{\dot{M}_{\mathrm{peb}}}{\dot{M}_{*}} .
\end{aligned}
$$

Now, the parameter $\Lambda$ may be estimated in the limit of a vertically isothermal disk as

$\Lambda^{-1}=1+\frac{\Sigma_{\mathrm{p}} / h_{\mathrm{p}}}{\Sigma_{\mathrm{g}} / h_{\mathrm{g}}}=1+Z \frac{h_{\mathrm{g}}}{h_{\mathrm{p}}}$.

This thus leads to the following second-order equation in $Z$ :

$\left(\xi \beta^{2}-\beta\right) Z^{2}-(A+1-2 \xi \beta) Z+\left(1+\tau_{s}^{2}\right) \xi=0$,

where we have defined a few quantities,

$A \equiv \frac{2 \tau_{\mathrm{s}}}{3 \alpha}\left(-\frac{\mathrm{d} \ln P}{\mathrm{~d} \ln r}\right) \equiv a_{0}\left(\tau_{\mathrm{s}} / \alpha\right)$,

and we adopt $a_{0} \approx 1.75$ as $a_{0}$ is estimated to be $\approx 1.70-1.85$ for radially smooth disks with both viscous heating and stellar irradiation (see Ida et al. 2016), $\beta$ is the ratio of the gas-to-dust pressure scale heights (Dubrulle et al. 1995; Youdin \& Lithwick 2007),

$\beta \equiv h_{\mathrm{g}} / h_{\mathrm{p}} \simeq\left(1+\tau_{\mathrm{s}} / \alpha\right)^{1 / 2}$,

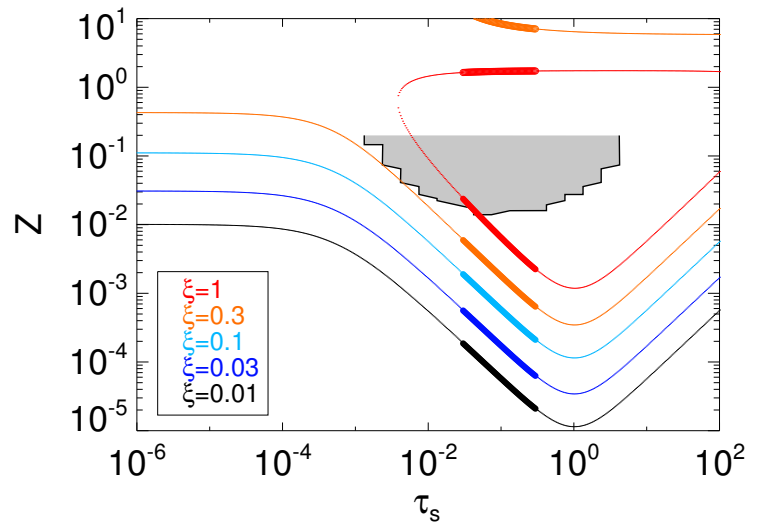

Fig. 1. Steady-state solutions for the solid-to-gas mixing ratio $Z$ as a function of the Stokes number of solid particles $\tau_{\mathrm{s}}$ for different values of the solid-to-dust-mass flux ratio $\xi$ (as labeled), assuming a value of the turbulent viscosity $\alpha=10^{-3}$. The values of $\tau_{\mathrm{s}}$ corresponding to expected pebble sizes are highlighted with larger symbols. The two solutions provided by Eq. (13) are indicated by filled and open symbols, respectively. The gray area highlights the region in which planetesimals should form by a streaming instability (Carrera et al. 2015).

and $\xi$ is the ratio of the solid mass flux to the gas mass flux:

$\xi \equiv \dot{M}_{\mathrm{peb}} / \dot{M}_{*}$.

The solutions to Eq. (9) are

$Z=\frac{2 \xi \beta-(A+1) \pm \sqrt{(A+1)^{2}-4 \xi \beta\left(A+\xi \beta \tau_{\mathrm{s}}^{2}-\tau_{\mathrm{s}}^{2}\right)}}{2 \beta(1-\xi \beta)}$.

Figure 1 shows the solutions to Eq. (13) obtained for $Z$ as a function of $\tau_{\mathrm{s}}$ for different values of the pebble-to-gas-mass flux ratio $\xi$. For pebbles, we expect $\tau_{\mathrm{s}} \sim 0.1$ (Sato et al. 2016; Ida et al. 2016), so that $\tau_{\mathrm{s}} \gg \alpha$ and hence $A / a_{0} \simeq \beta^{2} \gg 1$, yielding the approximate solution

$Z_{\mathrm{peb}} \sim \frac{\xi}{a_{0}} \alpha \frac{\tau_{\mathrm{s}}^{2}+1}{\tau_{\mathrm{s}}}$

which fits the lower- $Z$ solutions in Fig. 1 for $\tau_{\mathrm{s}} \gtrsim 10^{-2}$. This approximate solution can be easily derived from Eq. (7) with $\tau_{\mathrm{s}} \gg \alpha$ and $\Lambda \simeq 1$. The dependence on $\alpha$ appears because, as shown by Eq. (1), $\Sigma_{\mathrm{g}} \propto 1 / \alpha$ and $\Sigma_{\mathrm{p}}$ is independent of $\alpha$ for $\tau_{\mathrm{s}} \sim$ 0.1 . The fast drift of pebbles is responsible for the small $Z_{\mathrm{peb}}$.

We expect planetesimal formation to occur either by GI in the dust disk when $\rho_{\mathrm{p}} \gtrsim \rho_{\mathrm{R}}$, where $\rho_{\mathrm{R}} \sim M_{*} / r^{3}$ is the Roche density, independently of the dust size, or by SI with $Z$ as low as $\sim 0.02$ but a limited range of $\tau_{\mathrm{s}}$ values (Dra̧żkowska \& Dullemond 2014; Carrera et al. 2015, also see Fig. 1). In general, the condition for GI, $\rho_{\mathrm{p}} \gtrsim \rho_{\mathrm{R}}$, is difficult to reach because of the vertical shear that is due to $\mathrm{KH}$ instabilities and thus requires very high values of $Z$.

We see in Fig. 1 that the formation of pebbles by SI is possible but requires high values of $\xi$. The criterion for planetesimals to form directly from $\tau_{\mathrm{s}} \sim 0.1$ pebbles is $Z_{\text {pebble }} \gtrsim 0.02$. From Eq. (14), this implies

$\xi \gtrsim \xi_{\text {crit }, \mathrm{SI}} \equiv 3.5 / \alpha_{3}$,

where $\alpha_{3}=\alpha / 10^{-3}$. We show below that this condition, which requires the mass flux of pebbles to be equivalent to the mass flux of gas, is difficult to reach (see also Krijt et al. 2016). One possibility is to advocate high values of $\alpha$ (see Armitage et al. 2016), 
but this is generally not favored by the latest magnetohydrodynamical simulations of protoplanetary disks (e.g., Bai 2015). Other possibilities exist that require local perturbations in the disk to modify the pressure gradient term (e.g., Johansen et al. 2014, and references therein) or favorable conditions in terms of fragmentation threshold velocity and disk properties (Laibe 2014; Drazkowska et al. 2016). We show below that the conditions necessary to form planetesimals can be reached at lower $\xi$ values and for small $\alpha$-disks next to a snow line. Before we examine this possibility, it is worth noting that for small particles (with $\tau_{\mathrm{s}} \lessgtr 10^{-3}$ ), no solution is found for $\xi \gtrsim 1$, meaning that no steady-state exists: if there were a way to have small particles drift in at a very high rate or to deplete disk gas preferentially, the particles would pile up and accumulate, eventually forming planetesimals by direct gravitational instability.

\section{Solid-to-gas density ratio inside of the snow line}

When the inward-drifting pebbles cross the snow line, they progressively sublimate until only small refractory (silicate dust) seeds remain (e.g., Saito \& Sirono 2011; Morbidelli et al. 2016). Observations of the interstellar medium and of interplanetary dust particles indicate that these dust seeds should be of submicron size, corresponding to $\tau_{\mathrm{s}} \sim 10^{-7}-10^{-5}$. Even for the much larger millimeter-sized chondrules, we expect $\tau_{\mathrm{s}}$ to be between $10^{-4}$ and $5 \times 10^{-2}$ at most in a rarefied disk. The presence of a snow line is thus a way of transforming a high-mass flux of fastdrifting pebbles into a flux of small, slow-drifting dust particles.

Two additional factors need to be considered. First, the sublimation of the ice decreases the amount of solid material by a factor $\zeta_{0} \sim 1 / 3$ corresponding to the ratio of the mass of dust (silicate components) to the total mass of condensates (dust+ice) (Lodders 2003). By assuming for simplicity that the pebbles instantaneously form small dust particles, the flux of material to inside of the snow line that is to be considered in Eq. (13) is now therefore $\xi \rightarrow \zeta_{0} \xi_{\text {peb }}$, where $\xi_{\text {peb }}$ corresponds to the ratio of the non-sublimated pebble mass flux to the gas mass flux.

Second, we expect dust grains to retain a memory of the vertical scale height of the pebbles. Their vertical mixing timescale can be estimated to be

$t_{\text {mix }} \sim\left(h_{\mathrm{g}} / \ell\right)^{2} \Omega_{\mathrm{K}}^{-1} \sim 160 \alpha_{3}^{-1} T_{\mathrm{K}} \sim 160 \alpha_{3}^{-1}(r / 1 \mathrm{au})^{3 / 2} \mathrm{yr}$,

where $\ell \sim \sqrt{\alpha} h_{\mathrm{g}}$ is the estimated vertical mean free path and $T_{\mathrm{K}}$ is Kepler period. Comparing a sublimation timescale with a migration timescale for pebbles, we can derive the radial width for completion of the sublimation as $\Delta r \sim$ $10^{-2}(R / 10 \mathrm{~cm})^{1 / 2} r$ (see also Ciesla \& Cuzzi 2006). With Eq. (1), the timescale for the pebble flux to establish $Z \gtrsim 1$ in the sublimation region is estimated as $t_{Z} \sim 2 \pi r \Delta r \Sigma_{\mathrm{g}} / \dot{M}_{\mathrm{peb}} \sim$ $(1 / 3 \pi)\left(r / h_{\mathrm{g}}\right)^{2}(\Delta r / r) \alpha^{-1} \xi_{\mathrm{peb}}^{-1} T_{\mathrm{K}} \sim 10^{3}(R / 10 \mathrm{~cm})^{1 / 2} \alpha_{3}^{-1} \xi_{\mathrm{peb}}^{-1} T_{\mathrm{K}}$. Although $t_{Z}$ for $R=10 \mathrm{~cm}$ is $10-100$ times longer than $t_{\text {mix }}$, the effective $R$ for sublimation would be much smaller and $t_{Z}$ would be much shorter for more realistic fluffy pebbles (e.g., Kataoka et al. 2013). We can thus assume that the dust seeds released by the sublimating pebbles have the same vertical thickness as the pebbles themselves. This is done in Eq. (13) by replacing $\beta$ by the value set by the pebble subdisk $\beta \rightarrow \beta_{0} \sim$ $\left(1+\tau_{\mathrm{s}, \mathrm{peb}} / \alpha\right)^{1 / 2}$.

As shown in Fig. 2, the situation is now much more favorable toward the development of GI of the layer of sublimated pebbles. As pointed out by Saito \& Sirono (2011) and Birnstiel et al. (2012), the release of small slow-drifting dust by fast-drifting pebbles naturally yields values of $Z$ that are much higher than

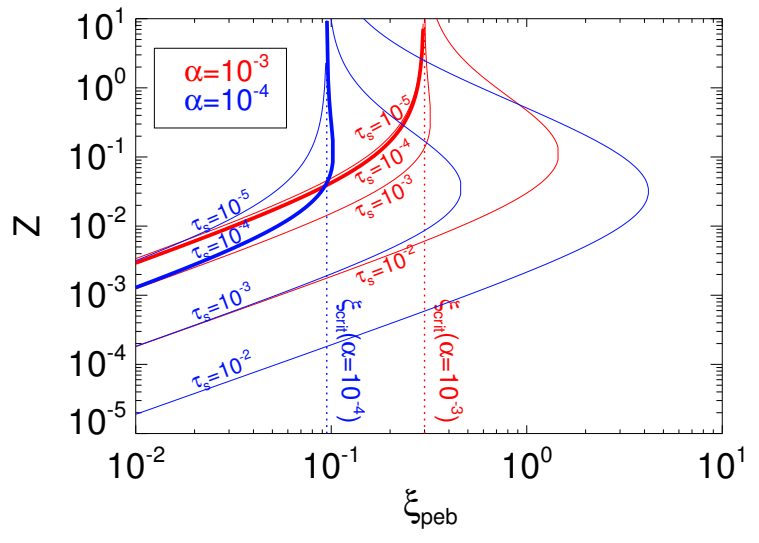

Fig. 2. Steady-state solutions for the solid-to-gas mixing ratio $Z$ as a function of the solid-to-gas-mass flux ratio $\xi$ for different values of the Stokes number of solid particles $\tau_{\mathrm{s}}$ (from $10^{-5}$ to $10^{-2}$, as labeled), assuming two values of the turbulent viscosity $\alpha=10^{-4}$ (in blue) and $\alpha=10^{-3}$ (in red). Equation (13) is numerically solved. In contrast to Fig. 1, we now consider that initially icy pebbles with $\tau_{\text {s,peb }} \sim 0.1$ and containing a mass fraction $\zeta_{0}=1 / 3$ in dust sublimate inside of the snow line. The thicker lines corresponds to the preferred value for the dust particles, $\tau_{\mathrm{s}}=10^{-4}$.

for pebbles. It first increases linearly with $\xi_{\text {peb }}$. However, when the pebble flux is high enough to yield $\rho_{\mathrm{p}} / \rho_{\mathrm{g}} \gtrsim 1$, the dust migration velocity decreases as a result of the inertia of the dust, which further enhances $\rho_{\mathrm{p}} / \rho_{\mathrm{g}}$. The value of $Z$ then increases more than linearly with $\xi_{\text {peb }}$ until the positive feedback becomes so strong that no steady-state solution can be found. At this point, we expect $\rho_{\mathrm{p}}$ to rapidly exceed $\rho_{\mathrm{R}}$, and planetesimals are formed by GI.

It is useful at this point to rederive Eq. (13) for these small grains, that is, in the limit of $A \ll 1$ and $\tau_{\mathrm{s}} \ll 1$ :

$Z \sim \frac{\zeta_{0} \xi_{\mathrm{peb}}}{1-\beta \zeta_{0} \xi_{\mathrm{peb}}}$

For these small grains, the critical value $\xi_{\text {crit }}$ above which no steady-state solution exists can be easily derived by calculating when the denominator of Eqs. (13) or (17) becomes zero, that is,

$\xi_{\text {crit }}=\frac{1}{\beta \zeta_{0}}=\frac{1}{\zeta_{0}\left(1+\tau_{\mathrm{s}, \mathrm{peb}} / \alpha\right)^{1 / 2}} \simeq \frac{1}{\zeta_{0}}\left(\frac{\alpha}{\tau_{\mathrm{s}, \mathrm{peb}}}\right)^{1 / 2}$.

The critical value $\xi_{\text {crit }}$ is thus independent of the local pressure gradient. This is because the small dust grains ejected from sublimating pebbles are coupled to the disk gas motion and migrate with disk gas accretion and not by gas drag.

As shown by Fig. 2, higher values of $\xi_{\text {crit }}$ are possible for $\tau_{\mathrm{s}} \gg \alpha$, a possibility that we do not consider here because we expect seed grains to be such that $\tau_{\mathrm{s}} \lesssim 10^{-5}$. Typically, we thus obtain $\xi_{\text {crit }} \simeq 0.3$ for $\alpha=10^{-3}, \zeta_{0}=1 / 3$ and $\tau_{\text {s,peb }}=0.1$. The fact that $\xi_{\text {crit }}$ scales with $\alpha^{1 / 2}$ is governed by the height of the dust and pebble subdisk. The formation of dust-rich planetesimals inside of the snow line is thus favored in weakly turbulent disks.

\section{Pebble flux and planetesimal formation}

The pebble mass flux is calculated by the mass in dust swept by the pebble formation front at $r \simeq r_{\text {peb }}$ per unit time (Lambrechts \& Johansen 2014; Ida et al. 2016),

$\dot{M}_{\mathrm{peb}} \simeq 2 \pi r_{\mathrm{peb}} \times Z_{0} \Sigma_{g}\left(r_{\mathrm{peb}}\right) \times \frac{\mathrm{d} r_{\mathrm{peb}}}{\mathrm{d} t}$, 
where $Z_{0}$ is the solid-to-gas ratio in the pebble formation region. The growth time of pebbles from $\mu \mathrm{m}$ sized dust grains is given by (Takeuchi \& Lin 2005; Okuzumi et al. 2012; Ida et al. 2016)

$t_{\text {grow }} \sim 10 \times \frac{4}{\sqrt{3 \pi}} \frac{1}{Z_{0} \Omega} \sim 210 Z_{02}^{-1} M_{* 0}^{-1 / 2}\left(\frac{r_{\text {peb }}}{1 \mathrm{au}}\right)^{3 / 2} \mathrm{yr}$,

where $Z_{02}=Z_{0} / 0.01$. From this equation, the pebble formation front radius is given by

$r_{\text {peb }} \simeq 290 Z_{02}^{2 / 3} M_{* 0}^{1 / 3} t_{6}^{2 / 3} \mathrm{au}$,

where $t_{6}=t / 10^{6} \mathrm{yr}$. Because ice needs to condense, $r_{\text {peb }} \gtrsim 1 \mathrm{au}$, implying that pebbles may start forming when $t \gtrsim 200 \mathrm{yr}$. Substituting this relation into Eq. (19) with $\dot{r}_{\text {peb }} / r_{\text {peb }}=\left(2 / 3 t_{\text {grow }}\right)$, we obtain

$$
\begin{aligned}
\xi_{\text {peb }, \mathrm{pf}} & =\frac{\dot{M}_{\text {peb }, \mathrm{pf}}}{\dot{M}_{*}}=\frac{\dot{M}_{\mathrm{peb}, \mathrm{pf}}}{3 \pi \Sigma_{\mathrm{g}} \alpha h_{\mathrm{g}}^{2} \Omega}=\frac{\sqrt{3 \pi}}{90} \frac{Z_{0}^{2}}{\alpha}\left(\frac{r_{\mathrm{peb}}}{h_{\mathrm{g}}}\right)^{2}, \\
& \simeq 0.30 L_{* 0}^{-2 / 7} M_{* 0} \alpha_{3}^{-1} Z_{02}^{34 / 21} t_{6}^{-8 / 21},
\end{aligned}
$$

where $L_{* 0}=L_{*} / L_{\odot}, M_{* 0}=L_{*} / M_{\odot}, \alpha_{3}=\alpha / 10^{-3}$, and we considered the irradiation-dominated regime with $T \simeq$ $120 L_{* 0}^{2 / 7} M_{* 0}^{-1 / 7}(r / 1 \mathrm{au})^{-3 / 7} \mathrm{~K}$, for the pebble formation region in the outer disk. This corresponds to the disk aspect ratio,

$\frac{h_{\mathrm{g}}}{r} \simeq 0.021 L_{* 0}^{1 / 7} M_{* 0}^{-4 / 7}\left(\frac{r}{1 \mathrm{au}}\right)^{2 / 7}$.

(The expressions are slightly simplified compared to Ida et al. 2016.) The pebble mass flux is given by $\xi_{\text {peb,pf }} \dot{M}_{*}$, implying

$\dot{M}_{\text {peb,pf }} \simeq 0.90 \times 10^{-3} L_{* 0}^{-2 / 7} M_{* 0} \alpha_{3}^{-1} Z_{02}^{5 / 3} \dot{M}_{* 8} t_{6}^{-1 / 3} M_{\oplus} / \mathrm{yr}$,

where $\dot{M}_{* 8}=\dot{M}_{*} /\left(10^{-8} M_{\odot} /\right.$ yr $)$. This mass flux is higher than obtained by Lambrechts \& Johansen (2014) because they assumed a lower initial surface density in the gas disk $\left(\Sigma_{\mathrm{g}}=\right.$ $500 \mathrm{~g} / \mathrm{cm}^{2}$ at $1 \mathrm{au}$, not tied to the mass flux in the disk) and a $1 / 2$ coagulation probability.

When $r_{\text {peb }}$ exceeds the disk size $r_{\text {out }}$, that is, when $t \gtrsim$ $2 \times 10^{5}\left(r_{\text {out }} / 100 \mathrm{au}\right)^{3 / 2} \mathrm{yr}$, we would expect $\dot{M}_{\text {peb }}$ to decay more rapidly than $\dot{M}_{*}$ because most of the solid material has been made into pebbles and drifted in (Sato et al. 2016). This may be inconsistent with the observational data that show that $\mathrm{mm}$ or $\mathrm{cm}$ sized particles survive in the disks for several million years (Brauer et al. 2007). However, we expect strong turbulence due to GI of the gas disk (not GI of the dust subdisk) to limit this fast spread of the pebble front, which could explain the presence of $\mathrm{mm}$ or $\mathrm{cm}$ sized particles for relatively long times.

Disks with $\Sigma_{\mathrm{g}}$ given by Eq. (1) are gravitationally unstable in their outer parts unless the disk is compact. In the unstable parts, their surface density should evolve to become marginally unstable so that $1 \sim Q=c_{\mathrm{s}} \Omega / \pi G \Sigma_{\mathrm{g}, \mathrm{GI}}$, or equivalently

$\Sigma_{\mathrm{g}, \mathrm{GI}} \simeq Q^{-1} \frac{h_{\mathrm{g}}}{r} \frac{M_{*}}{\pi r^{2}}$

where $Q$ is a factor on the order of unity. In these regions, the turbulence generated by gravitational waves (assumed to lead to $\alpha_{\mathrm{GI}} \sim 0.1$ ) may be high enough that collisions between icy grains result in fragmentation rather than coalescence. Typical collision velocities are estimated to be $v_{\mathrm{col}} \sim \sqrt{3 \alpha_{\mathrm{GI}} \tau_{\mathrm{s}}} c_{\mathrm{s}}$ (Sato et al. 2016). Assuming a sound speed $c_{\mathrm{s}} \sim 270(r / 100 \mathrm{au})^{-3 / 14} \mathrm{~m} / \mathrm{s}$ implies $v_{\text {col }} \sim 50\left(\alpha_{\mathrm{GI}} / 0.1\right)^{1 / 2}\left(\tau_{\mathrm{s}} / 0.1\right)^{1 / 2}(r / 100 \mathrm{au})^{-3 / 14} \mathrm{~m} / \mathrm{s}$. The threshold velocity for fragmentation of icy particles is predicted to be around 20-100 m/s (e.g., Blum \& Wurm 2000; Zsom et al. 2011; Wada et al. 2011). We therefore assume that pebbles may form only in the stable parts of the disks, that is, for $r<r_{\mathrm{GI}}$, implying that the location of the pebble formation front is given by $\min \left(r_{\mathrm{peb}}, r_{\mathrm{GI}}\right)$.

Since $\Sigma_{\mathrm{g}}$ given by Eq. (1) is equal to $\Sigma_{\mathrm{g}, \mathrm{GI}}$ at $r=r_{\mathrm{GI}}$, we obtain $Q\left(r_{\mathrm{GI}} / h_{\mathrm{g}}\right)^{3} \simeq 3 \alpha\left(M_{*} / \dot{M}_{*}\right) \Omega$, where $\alpha$ is the turbulence parameter for the inner regions. With Eq. (23),

$r_{\mathrm{GI}} \simeq 160 L_{* 0}^{2 / 3} M_{* 0}^{-1 / 3}\left(\alpha_{3} / Q \dot{M}_{* 8}\right)^{14 / 9} \mathrm{au}$.

As $\dot{M}_{*}$ decreases with time, $r_{\mathrm{GI}}$ increases. From $\left(\mathrm{d} r_{\mathrm{GI}} / \mathrm{d} t\right) / r_{\mathrm{GI}} \sim$ $-(14 / 9)\left(\mathrm{d} \dot{M}_{*} / \mathrm{d} t\right) / \dot{M}_{*}$ and Eq. (25), the pebble mass flux that is due to the outward spread of $r_{\mathrm{GI}}$ is

$$
\xi_{\mathrm{peb}, \mathrm{GI}}=\frac{2 \pi r_{\mathrm{GI}} \times Z_{0} \Sigma_{g} r_{\mathrm{GI}}}{\dot{M}_{*}} \simeq-\frac{14}{9} Z_{0} \frac{h_{\mathrm{g}}}{r_{\mathrm{GI}}} \frac{M_{*}}{\dot{M}_{*}^{2}} \frac{\mathrm{d} \dot{M}_{*}}{\mathrm{~d} t} .
$$

We now use the relation between accretion rate and age suggested by the observations of young clusters, $\dot{M}_{* 8} \sim t_{6}^{-3 / 2}$ (Hartmann et al. 1998). This yields

$r_{\mathrm{GI}} \simeq 160 L_{* 0}^{2 / 3} M_{* 0}^{-1 / 3}\left(\alpha_{3} / Q\right)^{14 / 9} t_{6}^{7 / 3} \mathrm{au}$

and

$\xi_{\text {peb,GI }} \simeq 0.24 L_{* 0}^{1 / 3} M_{* 0}^{1 / 3} Z_{02}\left(\alpha_{3} / Q\right)^{4 / 9} t_{6}^{7 / 6}$.

Equivalently,

$\dot{M}_{\mathrm{peb}, \mathrm{GI}} \simeq 0.79 \times 10^{-3} L_{* 0}^{1 / 3} M_{* 0}^{1 / 3} Z_{02}\left(\alpha_{3} / Q\right)^{14 / 9} t_{6}^{-1 / 3} M_{\oplus} / \mathrm{yr}$.

Figure 3 shows the evolution of the pebble formation front, the pebble mass flux $\dot{M}_{\text {per }}$, and $\xi_{\text {peb. The mass flux ratio } \xi_{\text {peb }}}$ reaches a maximum when $r_{\mathrm{GI}}=r_{\mathrm{peb}}$. This maximum value is proportional to $\alpha^{-29 / 45}$, whereas $\xi_{\text {crit }} \propto \alpha^{1 / 2}$. For the $\alpha=$ $10^{-3}$ case, we see that $\xi_{\text {peb }}$ becomes equal to $\xi_{\text {crit }}$ obtained from Eq. (18) only for a very short time. The $\alpha=10^{-4}$ case, in contrast, leads to a prolonged period in which planetesimals can form inside the snow line. Thus, direct formation of planetesimals is possible in low-turbulence disks. Conversely, in highturbulence disks, the relatively low maximum values of $\xi_{\text {peb }}$ obtained imply that other mechanisms have to be sought so that planetesimals can form. This may involve disk photoevaporation, disk winds, or growth of pebbles by ice condensation.

When formed by GI, the mass of a clump is $M_{\text {clump }} \sim h_{\mathrm{p}}^{3} \rho_{\mathrm{R}}$, where $\rho_{\mathrm{R}} \sim M_{*} / r^{3}$ is the Roche density. If the clump shrinks into a planetesimal with physical radius $R$ and bulk density $\rho_{s}\left(\sim 1 \mathrm{~g} \mathrm{~cm}^{-3}\right)$, then for the solar case,

$R \sim\left(\frac{h_{\mathrm{p}}}{r}\right)\left(\frac{M_{*}}{\rho_{s}}\right)^{1 / 3} \sim 10^{3}\left(\frac{h_{\mathrm{g}} / r}{0.01}\right) \alpha_{3}^{1 / 2}\left(\frac{\tau_{\mathrm{s}, \mathrm{peb}}}{0.1}\right)^{-1 / 2} \mathrm{~km}$.

Although a more detailed analysis would be required, it appears that the planetesimals formed through this mechanism are as large as those formed by SI (e.g., Johansen et al. 2014).

\section{Discussion and conclusion}

With a simple model of pebble growth, drift, and sublimation at the snow line, we have examined the conditions for the formation of planetesimals in protoplanetary disks.

We first showed that forming planetesimals from streaming instability in the flow of icy pebbles requires both a high level of turbulence $(\alpha \gtrsim 0.01)$ and an unrealistically high pebble flux. 

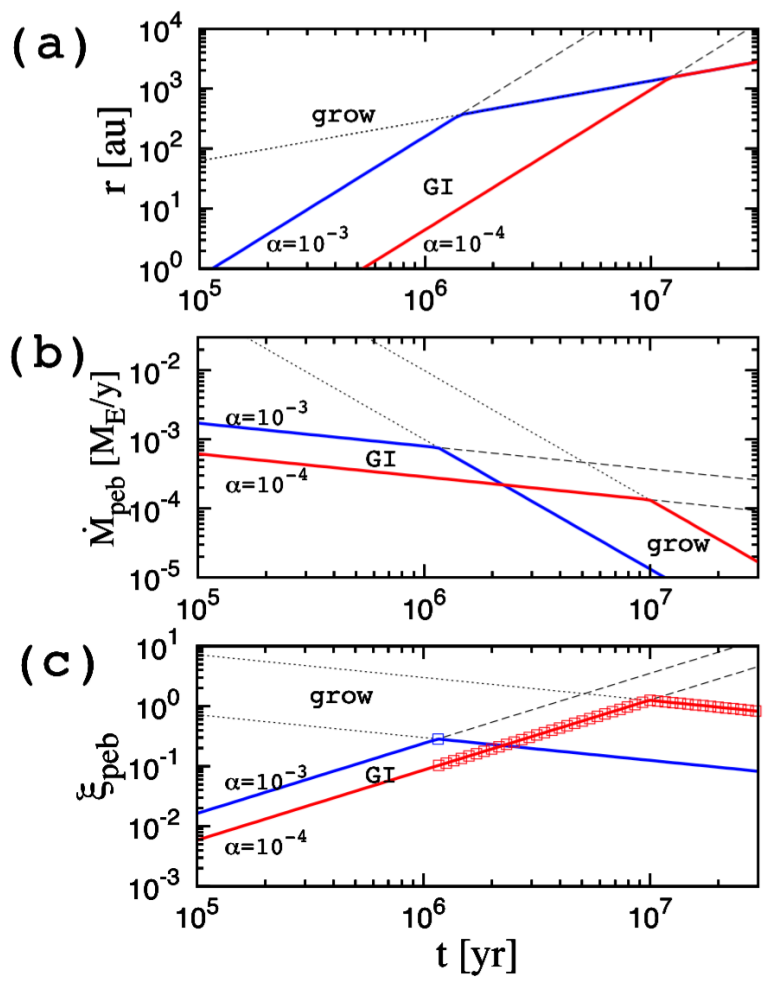

Fig. 3. Time evolution of a) radius of the pebble formation front, b) pebble accretion rate $\left(\dot{M}_{\mathrm{peb}}\right)$, and c) $\xi_{\mathrm{peb}}=\dot{M}_{\mathrm{peb}} / \dot{M}_{*}$ for two values of $\alpha$, $10^{-4}$ (red) and $10^{-3}$ (blue). The lines labeled "grow" (dotted) and "GI" (dashed) represent the pebble growth and disk GI limits, respectively. The thick solid lines express the actual values obtained by the minima of the two limits. Here we assumed $\tau_{\mathrm{s} \text {,peb }}=0.1$ and $Z_{0}=0.01$. In panel c), the small squares represent the points with $\xi_{\text {peb }}>\xi_{\text {crit }}$, see Eq. (18).

We note that for these high turbulence levels, water vapor diffusion can decrease the requirement on the pebble mass flux (e.g., Ros \& Johansen 2013; Armitage et al. 2016).

By including the often neglected mass-loading factor in the equations for the drift of solids, we have shown that the pile-up of solids inside of the snow line leads to the formation of dustrich planetesimals directly by gravitational instability in the dust subdisk. This instability exists for relatively high values of $\xi$, the pebble-to-gas-mass flux ratio, and for relatively low values of $\alpha$.

With a simple model of the formation of pebbles, we have demonstrated that super-critical values of the pebble-to-gas mass flux $\xi \geq \xi_{\text {crit }}$ are reached in disks with $\alpha \lesssim 10^{-3}$. This condition may be reached more easily, that is, for higher values of $\alpha$ or lower values of $\xi$, by taking into account disk gas depletion mechanisms other than viscous disk accretion such as photoevaporation or disk winds. The planetesimals that are formed in this way are expected to be large, probably larger than $100 \mathrm{~km}$ in radius.

During that time, the $\mathrm{H}_{2} \mathrm{O}$ snow line could move from several au to inside of 1 au (e.g., Oka et al. 2011). These planetesimals are expected to be composed of a high fraction of dust (silicates), which may explain why the rock-to-ice fractions inferred in minor planets and moons in the outer solar system (e.g., Schubert et al. 2010) or the dust-to-ice ratio in comets (e.g., Rotundi et al. 2015; Lorek et al. 2016) are often significantly higher than the expected $1 / 2$ to $1 / 3$ value obtained from purely solar composition (e.g., Lodders 2003).

Our model requires a fast breakup of pebbles, however, so that the dust particles are released over a small annulus. Given that we expect these pebbles to be porous (e.g., Kataoka et al. 2013), this should be verified. We note that the possibility that disks have flow in the midplane that are directed outward (Takeuchi \& Lin 2005) or are stochastic (Suzuki \& Inutsuka 2014) will favor the mechanism that we propose.

Finally, this process may apply to other sublimation lines (e.g., Drozdovskaya et al. 2016) if these lead to the breakup of fluffy pebbles into much smaller grains. Indeed, sintering has been shown to have this effect and thus might explain the rings that were recently observed in young disks (Okuzumi et al. 2016). The pile-up and planetesimal formation mechanism that we propose may thus naturally explain the formation of rings of planetesimals in low-turbulence disks.

Acknowledgements. We thank Chris Ormel, Anders Johansen, Satoshi Okuzumi, and an anonymous referee for helpful comments. S. I. thanks for the hospitality he experienced during his visit to the Observatoire de la Côte d'Azur, which was made possible thanks to support from $O C A B Q R$. S.I. is also supported by MEXT Kakenhi grant 15H02065. We acknowledge support by the French ANR, project number ANR-13-13-BS05-0003-01 projet MOJO (Modeling the Origin of JOvian planets).

\section{References}

Armitage, P. J., Eisner, J. A., \& Simon, J. B. 2016, ApJ, 828, L2 Bai, X.-N. 2015, ApJ, 798, 84

Birnstiel, T., Klahr, H., \& Ercolano, B. 2012, A\&A, 539, A148 Blum, J., \& Wurm, G. 2000, Icarus, 143, 138

Brauer, F., Dullemond, C. P., Johansen, A., et al. 2007, A\&A, 469, 1169

Carrera, D., Johansen, A., \& Davies, M. B. 2015, A\&A, 579, A43

Ciesla, F. J., \& Cuzzi, J. N. 2006, Icarus, 181, 178

Dra̧żkowska, J., \& Dullemond, C. P. 2014, A\&A, 572, A78

Drazkowska, J., Alibert, Y., \& Moore, B. 2016, A\&A, 594, A105

Drozdovskaya, M. N., Walsh, C., van Dishoeck, E. F., et al. 2016, MNRAS, 462, 977

Dubrulle, B., Morfill, G., \& Sterzik, M. 1995, Icarus, 114, 237

Goldreich, P., \& Ward, W. R. 1973, ApJ, 183, 1051

Guillot, T., Ida, S., \& Ormel, C. W. 2014, A\&A, 572, A72

Hartmann, L., Calvet, N., Gullbring, E., \& D'Alessio, P. 1998, ApJ, 495, 385

Ida, S., Guillot, T., \& Morbidelli, A. 2016, A\&A, 591, A72

Johansen, A., Oishi, J. S., Mac Low, M.-M., et al. 2007, Nature, 448, 1022

Johansen, A., Blum, J., Tanaka, H., et al. 2014, Protostars and Planets VI, 547

Kataoka, A., Tanaka, H., Okuzumi, S., \& Wada, K. 2013, A\&A, 557, L4

Krijt, S., Ormel, C. W., Dominik, C., \& Tielens, A. G. G. M. 2016, A\&A, 586, A20

Laibe, G. 2014, MNRAS, 437, 3037

Laibe, G., Gonzalez, J.-F., \& Maddison, S. T. 2012, A\&A, 537, A61

Lambrechts, M., \& Johansen, A. 2014, A\&A, 572, A107

Lodders, K. 2003, ApJ, 591, 1220

Lorek, S., Gundlach, B., Lacerda, P., \& Blum, J. 2016, A\&A, 587, A128

Morbidelli, A., Bitsch, B., Crida, A., et al. 2016, Icarus, 267, 368

Nakagawa, Y., Nakazawa, K., \& Hayashi, C. 1981, Icarus, 45, 517

Nakagawa, Y., Sekiya, M., \& Hayashi, C. 1986, Icarus, 67, 375

Oka, A., Nakamoto, T., \& Ida, S. 2011, ApJ, 738, 141

Okuzumi, S., Tanaka, H., Kobayashi, H., \& Wada, K. 2012, ApJ, 752, 106

Okuzumi, S., Momose, M., Sirono, S.-I., Kobayashi, H., \& Tanaka, H. 2016, ApJ, 821, 82

Ros, K., \& Johansen, A. 2013, A\&A, 552, A137

Rotundi, A., Sierks, H., Della Corte, V., et al. 2015, Science, 347, aaa3905 Saito, E., \& Sirono, S.-I. 2011, ApJ, 728, 20

Sato, T., Okuzumi, S., \& Ida, S. 2016, A\&A, 589, A15

Schubert, G., Hussmann, H., Lainey, V., et al. 2010, Space Sci. Rev., 153, 447

Sekiya, M. 1998, Icarus, 133, 298

Stevenson, D. J., \& Lunine, J. I. 1988, Icarus, 75, 146

Suzuki, T. K., \& Inutsuka, S.-i. 2014, ApJ, 784, 121

Takeuchi, T., \& Lin, D. N. C. 2005, ApJ, 623, 482

Wada, K., Tanaka, H., Suyama, T., Kimura, H., \& Yamamoto, T. 2011, ApJ, 737, 36

Weidenschilling, S. J. 1980, Icarus, 44, 172

Weidenschilling, S. J. 1995, Icarus, 116, 433

Youdin, A. N., \& Goodman, J. 2005, ApJ, 620, 459

Youdin, A. N., \& Lithwick, Y. 2007, Icarus, 192, 588

Youdin, A. N., \& Shu, F. H. 2002, ApJ, 580, 494

Zsom, A., Sándor, Z., \& Dullemond, C. P. 2011, A\&A, 527, A10 